\title{
Considerations for physicians using ketamine for sedation of children in emergency departments
}

\author{
Woo Sung Kim ${ }^{1}$, Ji Yeon Ku', Hanbyul Choi ${ }^{2}$, Hyo Jeong Choi', \\ Ho Jung Kim ${ }^{1}$, Bora Lee ${ }^{3}$ \\ 'Department of Emergency Medicine, Soonchunhyang University Bucheon Hospital, Bucheon, Korea \\ ${ }^{2}$ Department of Surgery, Stonybrook University Hospital, New York, USA \\ ${ }^{3}$ Department of Biostatistic Consulting, Clinical Trial Center, Bucheon Hospital of Soonchunhyang \\ University, Bucheon, Korea
}

Objective Ketamine use in emergency departments (EDs) for procedural sedation and analgesia is becoming increasingly common. However, few studies have examined patient factors related to adverse events associated with ketamine. This study investigated factors for consideration when using ketamine to sedate pediatric ED patients.

Methods The study included pediatric patients receiving ketamine for laceration repair in the ED. Before sedation, information was collected about upper respiratory tract infection symptoms, allergy history, and fasting time. Patients received $2 \mathrm{mg} / \mathrm{kg}$ ketamine intravenously or 4 $\mathrm{mg} / \mathrm{kg}$ ketamine intramuscularly. The primary outcomes were adverse events due to ketamine.

Results We studied 116 patients aged 8 months to 7 years (2.8 \pm 1.5 years). The group with adverse events was significantly younger on average than the group without adverse events ( $2.5 \pm 1.5$ vs. $3.1 \pm 1.5, P=0.028$ ). Upper respiratory tract infection symptoms were not significant variables affecting ketamine sedation (48.9\% vs. $43.7 \%, P=0.719$ ). There was no significant association between duration of fasting and adverse events $(P=0.073$ and $P=0.897$, respectively), or between food type and adverse events $(P=0.734)$. However, the number of attempts to sedate and ketamine dose correlated with adverse events $(\mathrm{P}<0.001$ and $\mathrm{P}=0.022$, respectively). In multiple logistic regression analysis, intravenous injection and ketamine dose were significant factors (odds ratio, $16.77 ; 95 \%$ confidence interval, 1.78 to 498.54 ; odds ratio, $4.37 ; 95 \%$ confidence interval, 1.59 to 22.9 , respectively).

Conclusion Emergency medicine physicians should consider injection type and ketamine dose when using ketamine sedation while suturing lacerations.

Keywords Emergency service, hospital; Ketamine; Pediatric sedation; Adverse events; Monitoring



eISSN: 2383-4625

Received: 10 July 2017

Revised: 21 September 2017

Accepted: 26 September 2017

Correspondence to: Ho Jung Kim Department of Emergency Medicine, Soonchunhyang University Bucheon Hospital, 170 Jomaru-ro, Wonmi-gu, Bucheon 14584, Korea

E-mail: lovelydr@schmc.ac.kr



How to cite this article:

Kim WS, Ku JY, Choi HB, Choi HJ, Kim HJ, Lee BR. Considerations for physicians using ketamine for sedation of children in emergency departments. Clin Exp Emerg Med 2017;4(4):244-249.

This is an Open Access article distributed under the terms of the Creative Commons Attribution Non-Commercial License (http:// creativecommons.org/licenses/by-nc/4.0/). 


\section{INTRODUCTION}

Patients in the emergency department (ED) often face extreme pain and anxiety from emergency procedures. To reduce their pain and anxiety, procedural sedation and analgesia are administered. Unlike general anesthesia, procedural sedation and analgesia achieve analgesia with sedation, such that patients can control their breathing on their own. In particular, pediatric trauma patients usually require sedatives. Although sedatives used in these cases are safe and have a short half-life, sedative use requires close monitoring because of potential serious adverse consequences that depend on the patient. However, monitoring itself is sometimes challenging in a busy ED.

Ketamine, a derivative of phencyclidine, is the most commonly used sedative and an ideal pediatric sedative in the ED., ${ }^{1,2}$ Oral sedatives, such as chloral hydrate, are also used, ${ }^{3}$ but they have unfavorable side effects, such as nausea, vomiting, agitation, restlessness, a longer recovery time, and high failure rates of up to $20 \% .{ }^{4}$ Ketamine inhibits the cerebral cortex and stimulates the limbic system, causing dissociation of the central nervous system from strong external stimuli. ${ }^{1,5,6}$ As a result, strong analgesia, sedation, or memory loss can be achieved using ketamine. However, ketamine also causes hypoxia, laryngospasm, apnea, bradycardia, hypersalivation, vomiting, and recovery agitation.?

One of the most worrying adverse events associated with sedatives is tracheal aspiration of regurgitated food; therefore, guidelines suggest that sedatives or analgesics should be administered on an empty stomach. ${ }^{8}$ However, although many institutions follow this guideline, several recent studies have reported that no association exists between preprocedural fasting state and adverse events from sedation. ${ }^{9,10}$ In addition, the clinical policy of the American College of Emergency Physicians published in 2014 recommended proceeding with sedation in the ED regardless of the fasting state, since preprocedural fasting does not reduce the risk of vomiting or aspiration. ${ }^{11}$ However, the American Academy of Pediatrics and American Society of Anesthesiologists guidelines suggest that the requirement for fasting before sedation depends on the type of food consumed and whether it is solid or liquid. ${ }^{8,12}$ However, the American Academy of Pediatrics guidelines also mentioned that the use of agents with less risk of depressing protective airway reflexes, such as ketamine, may be preferred for emergency patients. ${ }^{12}$

The purpose of this study was to analyze factors affecting adverse events related to ketamine administration in the pediatric population in the ED using a checklist and the establishment of key elements to consider in sedation using ketamine.

\section{METHODS}

\section{Study design and population}

This prospective observational study examined pediatric patients with simple lacerations requiring suturing from January to April 2016. Pediatric patients who would not co-operate with local anesthesia and who required sedation were included. All included patients were sedated using only ketamine. Patients were excluded for the following reasons: if they were younger than 3 months; had factors that elevated intraocular pressure (such as known glaucoma or acute globe injury) or intracranial pressure (such as known central nervous system masses, abnormalities, or hydrocephalus); had head trauma associated with loss of consciousness; had a previous allergic reaction to ketamine; or had parents who declined to participate in the study.

\section{Study protocol}

A standardized worksheet was created, and patient details pre- and post-ketamine administration were recorded. These included fasting duration, presence of upper respiratory tract infection symptoms (cough, runny nose, and sputum), allergic reaction, sedation time and frequency, vital signs, route of ketamine administration, adverse events, and procedures when adverse events occurred. The patients were divided into group with adverse events (GWAE) and group without adverse events (GWOAE). Patients with at least one adverse event were allocated to GWAE, and the remaining patients were allocated to GWOAE. To identify key elements related to adverse events, the factors of each group recorded in a checklist were compared. Adverse events included recovery agitation, involuntary movement, hypersalivation, vomiting, hypoxia, laryngospasm, cyanosis, apnea, and bradycardia. Hypoxia was defined as oxygen saturation $<95 \%$, and laryngospasm was characterized by stridor or retraction. Apnea was defined as the cessation of respiratory airflow. The length of time of cessation was 20 seconds or shorter if associated with bradycardia or cyanosis. Bradycardia was defined as a pulse below 60 beats/min (for a child older than two years) or a pulse below 80 beats/min (for a child younger than two years).

Subjects were closely monitored by one emergency medicine resident and one paramedic from the time of ketamine administration to complete recovery of consciousness, and an emergent airway management kit was made readily available next to the subjects. Resuscitative efforts included repositioning of the airway, oxygen administration and oropharyngeal airway insertion or intubation. All subjects were sedated with ketamine without local anesthesia for suturing, which was performed by a plastic surgeon.

All patients received $2 \mathrm{mg} / \mathrm{kg}$ ketamine intravenously (IV) or $4 \mathrm{mg} /$ kg ketamine intramuscularly (IM), according to the clinical situation. 
In the IV group, the initial dose was $2 \mathrm{mg} / \mathrm{kg}$ over 1 to 2 minutes under the supervision of an emergency medicine physician. If necessary, an additional $1 \mathrm{mg} / \mathrm{kg}$ was administered. In the IM group, the initial dose was $4 \mathrm{mg} / \mathrm{kg}$, followed by an additional $2 \mathrm{mg} / \mathrm{kg}$ if necessary.

Sedation induction time was defined as the time taken from administration to achieve appropriate sedation. The discharge criteria were as follows: appropriate oxygenation, alert to voice or light touch, stable airway during swallowing, and recovery of consciousness. The criteria for recovery of consciousness included age-appropriate language, recognition of parents/guardians, and purposeful movement, based on the Vancouver sedation scale. ${ }^{13}$

This study was approved by the institutional review board of Soonchunhyang University Hospital (2016-05-003-002), and the authors agreed on the study objective and protocol, and about procedural sedation and analgesia. The emergency medicine physician obtained consent from the parents/guardians of each patient.

\section{Analysis and statistics}

General characteristics of the study groups divided according to adverse events were summarized as mean \pm standard deviation for quantitative variables, and frequency (percentage) for qualitative variables. To compare the quantitative characteristics between the two groups, a Student's t-test or Mann-Whitney U-test was used as appropriate, according to the result of a normality and equivalent-variance test with Shapiro-Wilk test and Levene's test. A chisquare test was used to compare the qualitative variables.

Univariate logistic regression analysis was performed to examine the effect of candidate parameters on the occurrence of adverse events. For each parameter, the odds ratio $(O R)$ for the risk of the adverse event was expressed with a 95\% confidence interval $(\mathrm{Cl})$. For the multivariable logistic regression analysis, the Akaike information criterion-based backward selection method was used to exclude insignificant variables from the initial regression model, including factors considered statistically and clinically significant. Multiple collinearity of the continuous variables was assessed using generalized variance inflation factors.

A two-tailed P-value of less than 0.05 was considered statistically significant. All statistical analyses were conducted using SPSS ver. 14.0 (SPSS Inc., Chicago, IL, USA) and R ver. 3.1.3 (The $R$ Foundation for Statistical Computing, Vienna, Austria).

\section{RESULTS}

\section{Patient characteristics}

There were 116 subjects: 45 in the GWAE and 71 in the GWOAE. There were 87 in the IV group and 29 in the IM group. The mean patient age was $2.8 \pm 1.5$ years (range, 8 months to 7 years). The propor- tion of male subjects was $67.2 \%$. The mean procedure time (from appropriate sedation to completion of procedure) was $11.5 \pm 8.6$ minutes. The mean fasting duration was $3.8 \pm 1.7$ hours.

Age and number of trials to sedation differed statistically between GWAE and GWOAE $(2.5 \pm 1.5$ vs. $3.1 \pm 1.5$ years, $P=0.028$; $1.3 \pm 0.6$ vs. $1.0 \pm 0.1, P<0.001$, respectively). The ketamine dose $(\mathrm{mg} / \mathrm{kg})$ also differed significantly between the two groups (GWAE $2.9 \pm 1.1$ vs. GWOAE $2.5 \pm 0.9, P=0.022$ ) (Table 1 ).

Although there was no statistical difference, the procedure time was slightly longer in GWAE $(14.3 \pm 12.4$ vs. $9.8 \pm 4.3 \mathrm{~min}-$ utes, $P=0.341$ ) (Table 1).

\section{Adverse events according to presedation factors Univariate logistic regression analysis}

Three presedation factors were statistically related to adverse events: procedure time (minutes; $\mathrm{OR}, 1.07 ; 95 \% \mathrm{Cl}, 1.02$ to 1.14 ; $P=0.015)$; number of attempts required to sedate $(O R, 13.38$;

Table 1. Baseline characteristics according to adverse events

\begin{tabular}{|c|c|c|c|}
\hline Variable & GWAE $(n=45)$ & GWOAE $(n=71)$ & P-value \\
\hline \multicolumn{4}{|l|}{ Demographic factor } \\
\hline Age (yr) & $2.5 \pm 1.5$ & $3.1 \pm 1.5$ & 0.028 \\
\hline Sex & & & 0.922 \\
\hline Male & $31(68.9)$ & $47(66.2)$ & \\
\hline Female & $14(31.1)$ & 24 (33.8) & \\
\hline $\mathrm{BMI}\left(\mathrm{kg} / \mathrm{m}^{2}\right)$ & $16.6 \pm 3.9$ & $15.9 \pm 2.4$ & 0.359 \\
\hline \multicolumn{4}{|l|}{ ED arrival data } \\
\hline Body temperature $\left({ }^{\circ} \mathrm{C}\right)$ & $36.6 \pm 0.3$ & $36.6 \pm 0.4$ & 0.820 \\
\hline URI symptoms & $22(48.9)$ & $31(43.7)$ & 0.719 \\
\hline Type of food & & & 0.734 \\
\hline Solid & $25(55.6)$ & $43(60.6)$ & \\
\hline Liquid & $20(44.4)$ & $28(39.4)$ & \\
\hline Fasting duration (hr) & $3.5 \pm 1.7$ & $4.0 \pm 1.7$ & 0.073 \\
\hline Fasting duration (hr) & & & 0.897 \\
\hline Within criteria & $14(31.1)$ & $20(28.2)$ & \\
\hline Beyond criteria & $31(68.9)$ & $51(71.8)$ & \\
\hline \multicolumn{4}{|l|}{ Treatment to sedation } \\
\hline Procedure time (min) & $14.2 \pm 12.3$ & $9.8 \pm 4.4$ & 0.341 \\
\hline No. of attempts to sedate (n) & $1.3 \pm 0.6$ & $1.0 \pm 0.1$ & $<0.001$ \\
\hline Injection type of ketamine & & & 0.582 \\
\hline IV & $32(71.1)$ & $55(77.5)$ & \\
\hline IM & $13(28.9)$ & $16(22.5)$ & \\
\hline Dose of ketamine $(\mathrm{mg} / \mathrm{kg})$ & $2.9 \pm 1.1$ & $2.5 \pm 0.9$ & 0.022 \\
\hline
\end{tabular}

Data are presented as mean \pm standard deviation for continuous variables and frequency (\%) for categorical variables. P-values were calculated using a Student's t-test or Mann-Whitney U-test for continuous variables and chi-square test for categorical variables.

GWAE, group with adverse events; GWOAE, group without adverse events; BMI, body mass index; ED, emergency department; URI, upper respiratory infection; IV, intravenous; IM, intramuscular.

${ }^{a}$ Within criteria: fasting duration $\geq 2$ hours for liquid food and $\geq 6$ hours for solid food. 
Table 2. Logistic regression analysis for adverse events

\begin{tabular}{|c|c|c|c|c|}
\hline \multirow{2}{*}{ Variable } & \multicolumn{2}{|c|}{ Univariate analysis } & \multicolumn{2}{|c|}{ Multivariate analysis } \\
\hline & OR $(95 \% \mathrm{Cl})$ & P-value & OR $(95 \% \mathrm{Cl})$ & P-value \\
\hline Age $(y r)$ & $0.77(0.58-1.00)$ & 0.060 & & \\
\hline Female & $0.88(0.39-1.96)$ & 0.763 & & \\
\hline BMI $\left(\mathrm{kg} / \mathrm{m}^{2}\right)$ & $1.08(0.95-1.24)$ & 0.241 & & \\
\hline Body temperature $\left({ }^{\circ} \mathrm{C}\right)$ & $0.74(0.23-2.23)$ & 0.594 & & \\
\hline URI symptoms & $1.23(0.58-2.62)$ & 0.582 & & \\
\hline Solid food & $0.81(0.38-1.74)$ & 0.594 & & \\
\hline Fasting duration (hr) & $0.83(0.66-1.04)$ & 0.105 & $0.83(0.64-1.05)$ & 0.130 \\
\hline Fasting duration beyond criteria (hr) & $0.87(0.39-1.99)$ & 0.735 & & \\
\hline Procedure time (min) & $1.07(1.02-1.14)$ & 0.015 & & \\
\hline No. of attempts to sedate (n) & $13.38(2.83-243.79)$ & 0.012 & & \\
\hline IV injection & $0.72(0.31-1.70)$ & 0.442 & $16.77(1.78-498)$ & 0.034 \\
\hline Dose of ketamine $(\mathrm{mg} / \mathrm{kg})$ & $1.48(1.02-2.19)$ & 0.043 & $4.37(1.59-22.9)$ & 0.018 \\
\hline
\end{tabular}

$\mathrm{OR}$, odds ratio; $\mathrm{Cl}$, confidence interval; $\mathrm{BMI}$, body mass index; URI, upper respiratory infection; IV, intravenous.



Fig. 1. Proportion of adverse events.

95\% Cl, 2.83 to $243.79 ; \mathrm{P}=0.012)$; and dose of ketamine $(\mathrm{mg} / \mathrm{kg}$; $\mathrm{OR}, 1.48 ; 95 \% \mathrm{Cl}, 1.02$ to $2.19 ; \mathrm{P}=0.043)$. However, there were no significant associations between adverse events and age, URI symptoms, or fasting duration $(P=0.06, P=0.582$, and $P=0.735$, respectively) (Table 2 ).

\section{Multivariate logistic regression analysis}

Adverse events were significantly related to IV injection (OR, $16.77 ; 95 \% \mathrm{Cl}, 1.78$ to $498.54 ; \mathrm{P}=0.034)$ and ketamine dose $(\mathrm{OR}$, 4.37; $95 \% \mathrm{Cl}, 1.59$ to $22.9 ; \mathrm{P}=0.018$ ) (Table 2). In the multivariate logistic regression analysis, procedure time, number of attempts required to sedate, and fasting duration were not significantly related to adverse events.

\section{Proportion of adverse events}

The most frequent adverse event was involuntary movement (48.9\%). Other adverse events included recovery agitation (37.8\%), hypoxia (13.8\%), and hypersalivation (11.1\%) (Fig. 1).

\section{DISCUSSION}

Ketamine, which can be administered both IV and IM, is one of the most commonly used sedatives in pediatric cases. ${ }^{6}$ There are several key elements to consider when using ketamine for sedation in children; these are patient age, upper respiratory infection symptoms, injection route, and fasting period. Physicians using ketamine should consider the patient's condition and all factors related to the adverse events of ketamine. The most frequent adverse events observed in this study were involuntary movement and recovery agitation, and this is consistent with the results of other studies. ${ }^{14,15}$

This study enrolled pediatric patients. Therefore, we used the normal vital sign values in children that have been described in many previous studies. We defined hypoxia as an oxygen saturation $<95 \%{ }^{16}$ and bradycardia as a pulse rate $<60$ beats/min (for a child older than two years) or $<80$ beats/min (for a child younger than two years)..$^{17}$ Apnea was defined as the cessation of respiratory airflow for 20 seconds or less if associated with bradycardia or cyanosis. ${ }^{18}$

Previously published guidelines state that use of ketamine is relatively contraindicated in patients with lung diseases, such as asthma or upper respiratory infections. ${ }^{6}$ The current study, however, found no significant relationship between adverse events and upper respiratory tract infection symptoms $(P=0.582)$. If clinicians were to monitor $\mathrm{O}_{2}$ saturation in patients with upper respiratory tract infection symptoms more closely after administration of ketamine, the presence of such symptoms would not be a major concern with ketamine use.

This study found a statistically significant relationship between younger age and the likelihood of having an adverse event. How- 
ever, a recent study showed an increased rate of vomiting after ketamine use as patient age increased ( 7 vs. $>12$ years; $20.6 \%$ vs. $50 \%){ }^{19}$ In addition, other studies have shown a positive correlation between age and rate of adverse events from ketamine (8.2 vs. 5.2 years, $P=0.001$ ). ${ }^{9}$ In the current study, the age difference between the GWAE and the GWOAE was 0.6 years ( 7.2 months). This difference is statistically significant; however, the difference is small in clinical terms. A future study involving a larger patient sample with a broader age range would be beneficial for identifying a relationship between age and adverse events from ketamine.

This study also found no significant relationship between fasting duration and adverse events. We analyzed fasting duration beyond reported criteria. ${ }^{8,12}$ Another recent study found that fasting duration was significantly related to adverse events, especially vomiting, although hypoxia was not. ${ }^{10} \mathrm{~A}$ future study investigating the depth of sedation in more subjects would be valuable for understanding the relationship between fasting duration and adverse events.

Another study found that the likelihood of vomiting increased from $3.8 \%$ to $29 \%$ as fasting duration decreased..$^{20}$ In addition, it was reported that vomiting occurred after the procedure in up to $14 \%$ of cases, although no cases experienced vomiting in the middle of the procedure. ${ }^{19}$ In this study, there was only one case of vomiting $(<1 \%)$ during the procedure. A future study on the rate of ketamine-induced vomiting after discharge would add useful insight in clinical practice. However, based on recent evidence, ${ }^{9,19}$ physicians should perform the procedure regardless of the fasting period to provide shorter ED stays, effective flow management, and the prevention of overcrowding in the ED.

In this study, there was a significant difference in the rate of adverse events according to the route of administration. In the multivariate logistic regression analysis, the OR of IV ketamine was 16.77 ( $95 \% \mathrm{Cl}, 1.78$ to $498.54 ; \mathrm{P}=0.034)$. IV administration was also significantly related to adverse events of ketamine in other studies. ${ }^{4,15,21}$ In our series, the IV group was larger than the IM group (87 vs. 29 patients) and the $95 \% \mathrm{Cl}$ was too wide. In a future study, IM and IV groups with similar numbers may be required to obtain reliable results. Previous studies have demonstrated a sedation induction time of 30 to 60 seconds via the IV route and 3 to 5 minutes via the IM route. ${ }^{22,23}$ The current study found mean sedation induction times of 54 seconds (IV) and 4.6 minutes (IM), which are consistent with those described in the recent literature.

The subjects in this study were discharged to home without any serious adverse events. The minor adverse events in the current study were temporary and managed appropriately. One pre- vious study concluded that ketamine is a relatively safe sedative regardless of atropine use. ${ }^{24} \mathrm{~A}$ second study also reported no serious complications from sedative use in pediatric patients in the $E D$, with an adverse event rate of $2.3 \% .{ }^{25}$ In another study, no serious complications associated with ketamine administration at doses 5 to 100-fold higher than recommended (administered in error) were reported, except for minor temporary respiratory distress or prolonged sedation. ${ }^{26}$ Although this study found two cases of laryngospasm, they were mild in nature. According to current evidence, the rate of serious complications, such as laryngospasm or apnea, is $0.4 \%$ to $1.4 \% .^{21,27}$ In summary, ketamine is a safe sedative to use in pediatrics, with readily available resources for possible airway management.

This study focused on ketamine use only in the suturing of lacerations without local anesthesia. A comparison of ketamine versus local anesthesia during laceration suturing would advance our understanding of the choice of pain control. In addition, a further study on the effects of ketamine in different procedures related to dislocation, fractures, endoscopy, or foreign body removal would help clinical practices in minimizing adverse events.

The limitations of this study were the small sample size, the short duration of the study period, and the fact that researchers did not investigate all pediatric patients who presented to the ED with lacerations. Therefore, there was a possibility of selection bias.

This study was conducted until the end of the suturing procedure. The patients were monitored until discharge. There was no record of adverse events during the recovery period after sedation and discharge from the ED. More research is needed in a future study to address these limitations.

In summary, when administering ketamine sedation in ED pediatric patients undergoing suturing for lacerations, clinicians should consider the possibility of adverse events, which can be influenced by injection type and ketamine dose.

\section{CONFLICT OF INTEREST}

No potential conflict of interest relevant to this article was reported.

\section{REFERENCES}

1. Krauss $B$, Green SM. Procedural sedation and analgesia in children. Lancet 2006;367:766-80.

2. Roberts $J R$, Hedges $J R$, editors. Clinical procedures in emergency medicine. 4th ed. Philadelphia, PA: WB Saunders; 2004.

3. Jang $H Y$, Jung $J H$, Kyong $Y$, et al. Korean guidelines for pedi- 
atric procedural sedation and analgesia. J Korean Soc Emerg Med 2012;23:303-14.

4. Ko MJ, Choi JH, Cho YS, Lee JW, Lim H, Moon HJ. Is local anesthesia necessary in ketamine sedation for pediatric facial laceration repair? A double-blind, randomized, controlled study. J Trauma Inj 2014;27:178-85.

5. Krauss B, Green SM. Sedation and analgesia for procedures in children. N Engl J Med 2000;342:938-45.

6. Green SM, Krauss B. Clinical practice guideline for emergency department ketamine dissociative sedation in children. Ann Emerg Med 2004;44:460-71.

7. Lee $J S$, Jeon WC, Park EJ, et al. Adjunctive atropine versus metoclopramide: can we reduce ketamine-associated vomiting in young children? a prospective, randomized, open, controlled study. Acad Emerg Med 2012;19:1128-33.

8. American Society of Anesthesiologists Task Force on Sedation and Analgesia by Non-Anesthesiologists. Practice guidelines for sedation and analgesia by non-anesthesiologists. Anesthesiology 2002;96:1004-17.

9. Agrawal D, Manzi SF, Gupta R, Krauss B. Preprocedural fasting state and adverse events in children undergoing procedural sedation and analgesia in a pediatric emergency department. Ann Emerg Med 2003;42:636-46.

10. Teshome G, Braun JL, Lichenstein R. Ketamine sedation after administration of oral contrast: a retrospective cohort study. Hosp Pediatr 2015;5:495-500.

11. Godwin SA, Burton JH, Gerardo CJ, et al. Clinical policy: procedural sedation and analgesia in the emergency department. Ann Emerg Med 2014;63:247-58.

12. Cote CJ, Wilson S; American Academy of Pediatrics; American Academy of Pediatric Dentistry. Guidelines for monitoring and management of pediatric patients before, during, and after sedation for diagnostic and therapeutic procedures: update 2016. Pediatrics 2016;138:e20161212.

13. Macnab AJ, Levine M, Glick N, Phillips N, Susak L, Elliott M. The Vancouver sedative recovery scale for children: validation and reliability of scoring based on videotaped instruction. Can J Anaesth 1994:41:913-8.

14. Seo JP, Park JS, Hwang TS, Jang SJ, Kim SH. Ketamine use of pediatric sedation in emergency room. J Korean Soc Emerg Med 2000;11:339-44.
15. Choi JH, Cho YS, Kim HJ, Lee JW, Cho YS. Comparison of adverse events and satisfaction between iv and im routes for ketamine sedation in pediatric facial laceration repair in the emergency department. J Korean Soc Emerg Med 2012;23: 705-11.

16. Fouzas S, Priftis KN, Anthracopoulos MB. Pulse oximetry in pediatric practice. Pediatrics 2011;128:740-52.

17. Fleming $S$, Thompson $M$, Stevens $R$, et al. Normal ranges of heart rate and respiratory rate in children from birth to 18 years of age: a systematic review of observational studies. Lancet 2011;377:1011-8.

18. Nelson NM. Members of task force on prolonged apnea: reports of the task force on prolonged apnea of the American Academy of Pediatrics. Pediatrics 1978;61:651-2.

19. Treston G. Prolonged pre-procedure fasting time is unnecessary when using titrated intravenous ketamine for paediatric procedural sedation. Emerg Med Australas 2004;16:145-50.

20. Lee JS, Jeon WC, Park EJ, et al. Does ondansetron have an effect on intramuscular ketamine-associated vomiting in children? A prospective, randomised, open, controlled study. J Paediatr Child Health 2014;50:557-61.

21. Chong JH, Chew SP, Ang AS. Is prophylactic atropine necessary during ketamine sedation in children? J Paediatr Child Health 2013;49:309-12.

22. Pena BM, Krauss B. Adverse events of procedural sedation and analgesia in a pediatric emergency department. Ann Emerg Med 1999;34(4 Pt 1):483-91.

23. Green SM, Clark R, Hostetler MA, Cohen M, Carlson D, Rothrock SG. Inadvertent ketamine overdose in children: clinical manifestations and outcome. Ann Emerg Med 1999;34(4 Pt 1):492-7.

24. Holzman RS. Morbidity and mortality in pediatric anesthesia. Pediatr Clin North Am 1994;41:239-56.

25. Green SM, Rothrock SG, Lynch EL, et al. Intramuscular ketamine for pediatric sedation in the emergency department: safety profile in 1,022 cases. Ann Emerg Med 1998;31:68897.

26. Wills AJ, Stevens DL. Epilepsy in the accident and emergency department. Br J Hosp Med 1994;52:42-5.

27. Bergman SA. Ketamine: review of its pharmacology and its use in pediatric anesthesia. Anesth Prog 1999;46:10-20. 